\title{
Closeness degree algorithm and its application in load identification Yulan Shen \\ ${ }^{1}$ School of North China Electric Power University, Baoding 071000, China; 2351545956@qq.com
}

Keywords: closeness degree, Intelligent electric meter, load identification, steady state, transient state.

\begin{abstract}
In recent years, with the construction of smart grid, the participation degree of the demand side to the power market is increasingly high. For the identification of the load is like a list of electricity, can help the user side to better respond to peak and valley price, reasonable arrangement of electricity time, and then to achieve energy saving. This paper focuses on the analysis of household load identification, based on the data provided by smart meters, using the steady-state and transient power characteristics of household appliances as the reference value of the similarity comparison. By contacting the household electrical appliances manufacturers access to the main electrical equipment temporarily steady-state characteristics as the basic template, through closeness degree to determine the load and the working condition, by switching characteristics determine the electricity of load . The principle of this method is simple and easy to implement, and the experimental results verify the accuracy and feasibility of the method.
\end{abstract}

\section{Introduction}

With the development of smart grid, more and more sensing and monitoring device, intelligent instrument has been in power system, used to obtain real-time grid data.

At present, the implementation of load pattern recognition is more in some countries which have a high degree of automation and high degree of market power, China also continues to strengthen the research work in this area. Some countries have had preliminary load pattern extraction and recognition technology and software application Such as power companies in the United States, Brazil , Malaysia"s national power company also launched a load pattern extraction research and for large users of electricity inspection . Domestic grid data mining system research started relatively late. In terms of power user load pattern extraction ,a typical user load pattern recognition system has not been developed. In some institutions of higher learning and scientific research institutions, power customer load pattern extraction methods are studied and discussed only in theory . Therefore, according to the situation of our country, it is still a challenging problem to study and develop the mature power user load pattern recognition system. In the identification of the load, the main methods are: spectral clustering algorithm [1], transient characteristic quantity method [2], PMU measured data method of small disturbance [3] , but they all have some limitations, such as the recognition rate is low. The instantaneous eigenvalue method, less the amount of recognition and data acquisition processing work less, but its recognition accuracy is low compared with other methods ; the spectral clustering algorithm, roughing and careful selection of the identification of the distribution process can gradually narrow the range of recognition to improve the recognition accuracy, but brings the preliminary huge load clustering. In view of the above limitations, based on the characteristics of transient and steady state, we take the non intrusive load identification method to identify traditional household appliances.

\section{Basic principle}

Closeness is a measure of similarity between data objects, which is often used in pattern recognition, data analysis, image processing and so on. In general there are three methods to calculate the closeness: Hamming nearness, Euclidean closeness degree and lattice degree of nearness. These 
three calculation methods are not absolutely good or bad, depending on the actual situation. Euclidean distance method is simple and convenient, so the article uses it.

\section{European close degree[4]}

The Euclidean distance is used to calculate the closeness degree. 2 multidimensional vectors $\mathrm{X}$ and y can be obtained from the load waveform, and the Euclidean distance is:

$$
D(x, y)=\sqrt{\frac{1}{n} \sum_{i=1}^{n}\left(x_{i}-y_{i}\right)^{2}}
$$

The data are normalized.

$x_{i}^{\prime}=\frac{x_{i}}{a}, y_{i}^{\prime}=\frac{y_{i}}{b}$

In the type, $a=\max x, b=\max y, D(x, y) \in[0,1]$.

The more close to 1 , the greater the difference; otherwise, the smaller the difference.

So get closeness degree

$$
N(x, y)=1-D(x, y)=1-\sqrt{\frac{1}{n} \sum_{i=1}^{n}\left(x_{i}^{\prime}-y_{i}^{\prime}\right)^{2}}
$$

Type (2) only compare the curve on the trend, not to consider the amplitude. In order to make the proximity between the amplitude as same as the range of the variation range, the definition of the closeness between amplitude is defined as

$$
N_{a}^{b}=e^{-(a / b-1)^{2}}
$$

\section{Maximum membership degree principle}

According to the principle of the maximum membership degree, the load to be identified is incorporated into the load class in the database.

The principle of the maximum membership degree is defined as follows [5].

A standard template library is composed of m fuzzy subsets $A_{1}, A_{2}, \cdots, A_{m}$ on the domain u. If any of the elements $u_{0} \in U, \mu_{A_{i}}=\max \left(\mu_{A_{1}}\left(u_{0}\right), \mu_{A_{2}}\left(u_{0}\right), \cdots, \mu_{A_{m}}\left(u_{0}\right)\right), u_{0}$ is considered to belong to $A_{i}$.

The degree of closeness is used as the membership function, and the expression of the comprehensive membership degree vector is obtained:

$W$ for weight, $R$ for Membership degree matrix,

$L=\left[\mu_{A_{1}}\left(u_{0}\right), \mu_{A_{2}}\left(u_{0}\right), \cdots, \mu_{A_{m}}\left(u_{0}\right)\right]=W \times R$

$R=\left[N_{1}, N_{2}, \cdots N_{n}\right]$.

\section{Load identification}

\section{Steady state feature extraction of electrical equipment}

In order to make the comparison of the measured curve and the curve of the template, the load type is determined according to the principle of maximum membership degree, we need to extract the characteristic of the curve. In this section, we extract the steady state parameters[6], which are the steady state power amplitude, the steady state reactive power amplitude. To the resistance of different loads, there is obvious difference between the two steady state characteristics, and this feature can distinguish them; but for resistance inductive and resistive capacitive load, you will need to do further research. 


\section{Transient feature extraction of power equipment [7]}

According to the principle of electromagnetic induction, the inductor has a blocking effect on the current, and the transient characteristics of the inductive device is closely related to its opening and closing, which has been described in the relevant literature. The transient characteristics of household appliances are unique, which can be distinguished from other electrical appliances, especially for the same power resistance equipment. Therefore, we use the rising edge and falling edge of the maximum instantaneous change value of the electrical equipment $P_{\text {on }} 、 Q_{o n} 、 P_{\text {off }} 、 Q_{\text {off }}$, active and reactive power waveform vector $P_{w a v} 、 Q_{w a v}$, to describe its transient, the TV as an example, as shown in figure 2.

TV opening process:
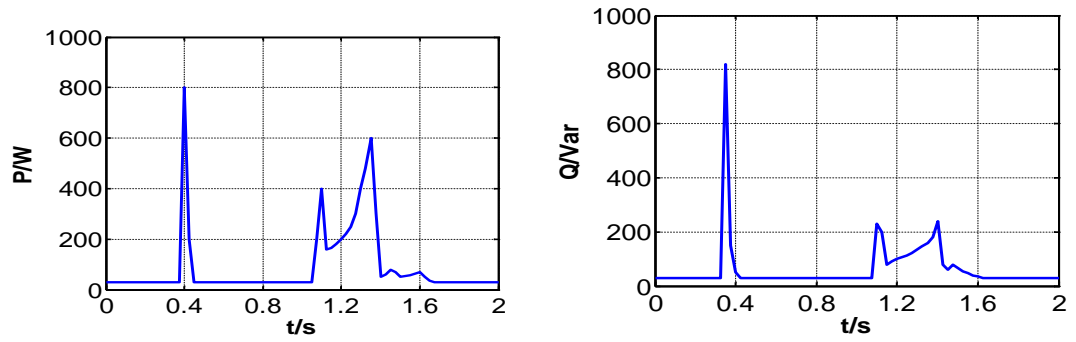

Fig. 1 power chart of TV opening process

TV closing process:
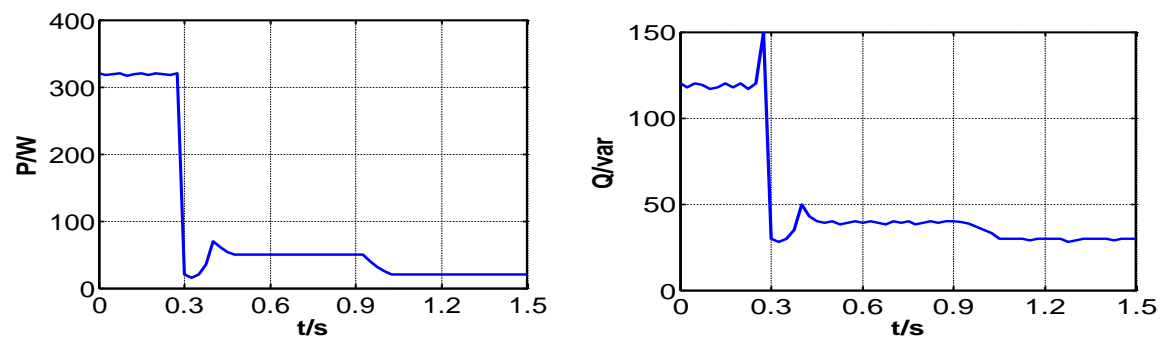

Fig. 2 power chart of TV closing process

Found from the measured data, the switching transient characteristics of different resistance load has obvious difference. As the transient time of this kind of refrigerator driven by a motor pump electric appliances is longer, the transient process of this kind of computer electronic equipment is very short, but the amplitude is very high.

For steady state characteristic $P_{\text {wen }} 、 Q_{\text {wen }}$, use the formula (3) to calculate the degree of closeness due to the magnitude of the relevant. For transient characteristic $P_{\text {on }} 、 Q_{o n} 、 P_{\text {off }} 、 Q_{\text {off }}$, use the formula (3) to calculate the degree of closeness. For $P_{w a v} 、 Q_{w a v}$ independent of amplitude, use the formula (2) to calculate the degree of closeness. Finally, according to the formula (4), the comprehensive membership degree is obtained.

\section{Summary}

This paper uses the closeness degree algorithm, simple and convenient, easy to achieve.

This paper selects residential electricity which is simple relatively in smart grid structure as the object of study, combines steady and transient characteristics of power , through establishing the database template in advance, and then compare the closeness degree of unknown load and household appliances template to determine which category, work status, and the electricity consumption the load belongs to.

Non intrusive load identification method directly obtain the required power parameters in the total interface, without affecting the accuracy of the data acquisition, greatly reducing the workload of data acquisition, and therefore easy to implement. 


\section{References}

[1] Wang Qingyu, Zhang Qingqing, Zhang Gaofeng, et al. Application of spectral clustering algorithm in household load identification[J]. Electrical Measurement \& Instrumentation, 2015,52(1):119-123.

[2] Qu Zhangyang, Yu huatao, Guo Xiaoli. Load identification of household appliances based on the characteristics of instantaneous load[J]. Transactions of China Electrotechnical Society, 2015,S1:358-364.

[3] Ma Ping, Wang Maohai, Wu Xinzhen, et al. Load parameter identification method based on small disturbance data measured by PMU[J]. Automation of electric power system, 2016,02:43-48

[4] Liu Fagui, Zhao Juan. Fuzzy closeness degree and its application[J]. Journal of North China Institute of water conservancy and hydroelectric power, 2006,03:104-106

[5] Li Honggi, Fuzzy mathematics foundation and practical algorithm[M]. Beijing: Science Press,2004

[6] Li Jing, Yang Honggeng. Load identification method for household intelligent electric meter[J]. Modern Electric Power, 2013， 30(5):83-89.

[7] Gao Yun, Yang Honggeng. Household load identification based on closeness degree matching of transient characteristics[J]. Automation of electric power system, 2013,37(9):54-59. 